\title{
OCLUSÃO VASCULAR E SUA APLICABILIDADE NA REABILITAÇÃO MUSCULOESQUELÉTICA: UMA ÁREA PROMISSORA
}

Aumentos da força muscular dependem de uma dose resposta adequada. Rhea et al. (2003) em uma meta-análise, concluíram que para indivíduos não treinados a intensidade de treinamento ótima para ganhos de força muscular era de $60 \%$ de 1RM (equivalente a 12 repetições máximas), ao passo que, para indivíduos treinados era de $80 \%$ de 1 RM (equivalente a 8 repetições máximas). Para os indivíduos idosos, recomenda-se uma intensidade entre 70-79\% de 1RM (BORDE; HORTOBÁGYI; GRANACHER, 2015). Porém, cargas equivalentes a $60-79 \%$ de 1RM podem impor riscos desnecessários e não são aplicáveis aos idosos frágeis ou pacientes pós-cirurgia, que possuem limitada capacidade física.

Considerando que o treinamento resistido com oclusão vascular impõe menor sobrecarga sobre as articulaçóes quando comparado com o treinamento resistido de alta intensidade. Possui aplicabilidade promissora em idosos com fraqueza muscular, artrite e outras comorbidades ortopédicas.

Baseado nessa informação e considerando que o método oclusão vascular pode ser uma medida preventiva contra a atrofia muscular causada pela desuso (COOK et al., 2010), Cook et al. (2017) compararam os efeitos do treinamento resistido de alta intensidade (70\% de 1RM) com o treinamento resistido de baixa intensidade e com oclusão vascular (30\% 1RM) em idosos com risco para limitação funcional. Os resultados desse estudo demonstraram que após 12 semanas de treinamento, ganhos de força muscular e hipertrofia foram similares entres os grupos. 
Takarada, Takazawa e Ishii (2000) avaliaram o feito do método oclusão vascular sobre os músculos da coxa em pacientes submetidos a cirurgia de reconstrução do ligamento cruzado anterior (LCA). O intuito do estudo foi saber se o método diminuiria o processo de atrofia muscular quando comparado com o grupo de pacientes que não utilizou o método oclusão vascular 13 dias após a cirurgia de LCA.

O método foi aplicado 2 vezes ao dia e consistia em 5 séries de oclusão vascular mantida por 5 minutos e 3 minutos de intervalo de recuperação sem oclusão vascular entre as séries (TAKARADA; TAKAZAWA; ISHII, 2000). Os resultados surpreendentes desse estudo demonstraram que o grupo controle, a área de secção transversa dos extensores e flexores do joelho diminuiu cerca de $20 \%$ e $11 \%$ respectivamente, enquanto que o grupo que realizou o método oclusão vascular, a área de secção transversa diminuiu somente $9 \%$ nos extensores e nos flexores do joelho, demonstrando que o método oclusão vascular aplicado 1 dia após a cirurgia de LCA durante 13 dias seguidos, foi capaz de retardar o processo de atrofia causado pelo desuso muscular pós-cirurgia.

Apesar desse resultado promissor, Iversen, Røstad e Larmo (2016) verificaram que o mesmo protocolo utilizado em uma população de atletas após 14 dias da cirurgia de LCA, não foi capaz de diminuir a atrofia dos extensores de joelho quando comparado ao grupo controle. Devemos considerar que no estudo de Takarada, Takazawa e Ishii (2000) os indivíduos permaneceram no hospital durante os 13 dias após a cirurgia, enquanto que no estudo de Iversen, Røstad e Larmo (2016) os pacientes realizaram o protocolo em casa.

Considerando que a supervisão direta tem uma implicação importante sobre a melhoria do aluno (GENTIL; BOTTARO, 2000; MAZZETI et al., 2000), é importante inferir que o método do estudo de Takarada, Takazawa e Ishii (2000) foi melhor controlado e como consequência obteve melhores resultados.

$\mathrm{Na}$ mesma linha de pesquisa, Ohta et al. (2003) avaliaram o efeito da reabilitação muscular realizada com oclusão vascular após a cirurgia de LCA. Os indivíduos foram separados em fisioterapia tradicional (sem oclusão vascular) e fisioterapia com oclusão vascular. A força muscular dos extensores e flexores de joelho foi avaliada antes e após 16 semanas da cirurgia, juntamente com a biópsia muscular para fibras do tipo I e II. Embora, sem diferenças entre os grupos, o grupo de indivíduos que realizou a fisioterapia com oclusão vascular apresentou menor declínio da força muscular dos extensores e flexores de joelho após 16 semanas da 
cirurgia de LCA. Além disso, o diâmetro das fibras tipo I e tipo II foram superiores no grupo que realizou a fisioterapia com a oclusão vascular quando comparados com o grupo que realizou a fisioterapia tradicional.

O programa de reabilitação ou fisioterapia realizado com oclusão vascular após a cirurgia de LCA (OHTA et al., 2003; TAKARADA; TAKAZAWA; ISHII, 2000) pode ser adaptado em situações práticas. A seguir o detalhamento de uma sugestão de um programa de reabilitação com oclusão vascular baseado em evidências:

- Um dia após a cirurgia de LCA, durante 13 dias seguidos realizar 5 séries de oclusão vascular por 5 minutos, 3 minutos de intervalo de recuperação sem oclusão vascular entre as séries e 2 vezes por dia (TAKARADA; TAKAZAWA; ISHII, 2000).

- Elevação das pernas e adução do quadril mantida por 5 segundos e repetida por 20 vezes - duas séries por dia - 6 vezes por semana durante as semanas 1-8. Após a cirurgia (um dia após a cirurgia até a primeira semana sem nenhuma carga); Semanas 2-4, uma carga com $1 \mathrm{~kg}$ foi adicionada aos pés; Semanas 5-8 após a cirurgia, uma carga de $2 \mathrm{~kg}$ foi adicionado (OHTA et al., 2003).

- A adução do quadril foi feita com uma bola utilizada na fisioterapia entre os joelhos -2 séries por dia -6 vezes por semana durante as semanas 1-12 após a cirurgia (OHTA et al., 2003).

- Agachamento parcial foi realizado por 6 segundos, repetido por 20 vezes. Step-up foi realizado subindo e descendo um step de $25 \mathrm{~cm}$ de altura repetido por 20 vezes (OHTA et al., 2003).

- O agachamento parcial foi realizado 2 séries por dia e o exercício de step-up foi realizado 3 vezes por dia durante 6 vezes por semana durante as semanas 5-16 após a cirurgia (durante as semanas 5-6, nenhuma carga; semanas 7-8 após a cirurgia, carga de 4 a $6 \mathrm{~kg}$ para deve ser sustentada pelas mãos; semanas 9-12 após a cirurgia, carga de 8 a $10 \mathrm{~kg}$, semanas 13-16 após a cirurgia, 12 a $14 \mathrm{~kg}$ ) (OHTA et al., 2003).

- Exercícios com elástico foram realizados com movimentos de flexão dos joelhos entre os ângulos 45 e $100^{\circ}$, repetidos por 20 vezes. Realizado com 1 série por dia, 6 vezes por semana durante as semanas 9-12 após a cirurgia e duas séries por dia durante as semanas 13-16 (OHTA et al., 2003).

- Para reabilitação do ligamento cruzado posterior (LCP) usar o método oclusão vascular juntamente com os exercícios wall squat short e one-leg squat, pois apresentam menor tensão sobre o LCP nesses exercícios quando comparado com o wall squat long. Além disso, lembrar que existe maior tensão sobre o LCP entre os ângulos de 80 - 90 (ESCAMILLA et al., 2009). 
- Para o LCA a maior tensão é encontrada no exercício one-leg squat (ESCAMILLA et al., 2009).

- Exercícios de cadeia cinética fechada como o leg press e squat apresentam co-contração dos isquiotibiais e quadríceps e isso é importante para minimizar o estresse sobre o LCA. Usar o método oclusão vascular juntamente com esses exercícios (ESCAMILLA et al., 2009).

- Lembrar que a tensão sobre o LCP é maior no exercício squat (ESCAMILLA et al., 2009).

- Para o LCA a força de tensão sobre ele na cadeira extensora é duas vezes maior quando a resistência é colocada perto do tornozelo (ESCAMILLA et al., 2012).

- Pode usar a oclusão vascular juntamente com a bicicleta ergométrica em pacientes pós-cirurgia de LCA (ESCAMILLA et al., 2012).

- Nos primeiros meses após a cirurgia de LCA o enxerto e sua fixação são significativamente fracos. Esperar cerca de 8 a 12 semanas para o processo adequado de ligamentização antes de introduzir cargas (ESCAMILLA et al., 2012).

- Verificar se os pacientes receberam profilaxia anticoagulante em grandes cirurgias, pois o risco de trombose pós-operação aumenta (DE BASTOS et al., 2001; LÓPEZ; KEARON; LEE, 2004).

O uso da fisioterapia com oclusão vascular também foi realizado em pacientes pós-cirurgia de artroscopia de joelho. Em um estudo aleatório controlado, realizado por Tennent et al. (2017), pacientes foram distribuídos aleatoriamente em dois grupos (fisioterapia tradicional e fisioterapia com oclusão vascular). Duas semanas após a cirurgia e liberação de seu cirurgião, passaram por 12 sessões de fisioterapia supervisionada durante 6 semanas. Os resultados do estudo demonstraram que o grupo que realizou a fisioterapia com oclusão vascular $(30 \%$ de $1 \mathrm{RM}, 4$ séries de 30/15/15/15 repetiçōes) quando comparado ao grupo que realizou a terapia tradicional, apresentou aumentos superiores na força muscular (extensores e flexores), hipertrofia muscular (circunferência da coxa) e funcionalidade.

Yow et al. (YOW et al., 2008) comentaram que os esforços tradicionais da reabilitação normalmente resultam em retorno incompleto para a função que o indivíduo deseja exercer. Com isso, o uso da reabilitação juntamente com a oclusão vascular permitirá uma recuperação em menor tempo, aumento da força muscular e melhoria da funcionalidade, considerando que os tecidos requerem tempo para cicatrização adequada antes que cargas sejam impostas com segurança (YOW et al., 2008). A prática tradicional da reabilitação foca na mobilização precoce, 
aumento progressivo da força, melhoria da amplitude de movimento durante os exercícios e não aplicação ou regulação rigorosa do estresse mecânico deletério no tecido que ainda está em processo de cicatrização (YOW et al., 2008).

Considerando que a força muscular do quadríceps, diâmetro das fibras tipo I e II, infiltração de gordura e área de secção transversa podem ficar prejudicadas 1 ano ou 48 meses após a cirurgia de LCA quando comparado com o membro não operado e que a resolução dos fatores após a cirurgia de LCA somente acontece após 2 anos (ARANGIO et al., 1997; LOPRESTI et al., 1998; NAGELLI; HEWETT, 2017). O método oclusão vascular sendo aplicado desde o início representa uma área promissora até para o retorno antecipado e com segurança as atividades cotidianas.

Considerando que o método oclusão vascular usando baixas cargas (20 a 30\% 1 RM) apresenta ganhos de força e hipertrofia similares ao treinamento resistido de alta intensidade ( $\geq 60 \%$ de $1 \mathrm{RM}$ ), o seu uso torna-se importante até em rupturas do tendão calcâneo utilizando o mesmo protocolo do estudo de Tennent et al. (2017) (30\% de 1RM, 4 séries de 30/15/15/15 repetições), apresentando resultados surpreendentes (YOW et al., 2018).

A caminhada lenta com oclusão vascular tem demonstrando importantes efeitos positivos sobre as respostas hormonais $(\mathrm{GH})$, hipertrofia e força muscular quando comparado com a caminhada sem oclusão vascular após 3 semanas de intervenção. Além disso, marcadores sanguíneos de dano muscular (atividade CPK e mioglobina) não foram elevados após a aplicação da caminhada lenta com oclusão vascular (ABE; KEARNS; SATO, 2006).

A dose resposta da frequência de treinamento também influencia as respostas hipertróficas e ganhos de força muscular, sendo superior para caminhada lenta com oclusão vascular realizada 2 vezes por dia e 6 vezes por semana (ABE; KEARNS; SATO, 2006) quando comparado com 1 por dia e 6 vezes por semana (ABE et al., 2009).

Esses dados sugerem que ganhos significativos de hipertrofia e força muscular podem ser alcançados sem danos musculares pela prática de exercícios de baixa intensidade e com oclusão vascular (ABE; KEARNS; SATO, 2006). Com isso, o método oclusão vascular pode ser benéfico para populações específicas, incluindo idosos, ou indivíduos debilitados que não podem tolerar exercícios com alta intensidade ou carga (COOK et al., 2017; BRYK et al., 2016; OHTA et al., 2003; TAKARADA; TAKAZAWA; ISHII, 2000; YASUDA et al., 2017). 
Bryk et al. (2016) demonstraram em um estudo aleatório controlado que o treinamento resistido de baixa intensidade ( $30 \%$ de $1 \mathrm{RM})$ quando comparado com o método tradicional ( $70 \%$ de $1 \mathrm{RM})$ induziu melhorias similares sobre a força muscular do quadríceps, funcionalidade e declínio da dor em pacientes mulheres com osteoartrite e idade média de 62 anos após 6 semanas de treinamento. Além disso, pacientes no grupo com oclusão vascular apresentaram dor inferior na parte anterior do joelho quando comparado com o método tradicional. Ferraz et al. (2017) também demonstraram efeitos similares entre o treinamento tradicional ( $80 \%$ de $1 \mathrm{RM}$ ) quando comparado com o treinamento resistido de baixa intensidade e com oclusão vascular ( $30 \%$ de 1 RM) no aumento da força muscular, hipertrofia, funcionalidade e dor em mulheres idosas com osteoartrite. Outro fator importante do estudo foram as desistências no treinamento tradicional devido a dor durante o treinamento. Demonstrando que menor estresse sobre as articulações e menor dor foram importantes para maior aderência no treinamento com oclusão vascular.

Sobrecarregar uma articulação com danos estruturais com um percentual alto de carga ( $>60 \%$ de $1 \mathrm{RM}$ ) não será tolerado e possivelmente induzirá lesões em um indivíduo que já possui dores no joelho ao realizar as atividades do cotidiano. Devido a baixa tolerância e ao alto risco conferido com carga altas, existe a necessidade para formas alternativas de treinamento (SEGAL et al., 2015).

Com isso, Segal et al. (2015) em um estudo duplo cego e aleatório controlado verificaram que o treinamento resistido de baixa de intensidade ( $30 \%$ de $1 \mathrm{RM})$ e com oclusão vascular após 4 semanas foi suficiente em aumentar a força muscular, potência e sem exacerbar as dores no joelho em mulheres com idade entre 45 e 65 anos e risco para osteoartrite assintomático, apesar de resultados controversos em homens com risco para osteartrite do mesmo grupo de pesquisa (SEGAL; DAVIS; MIKESKY, 2015).

Giles et al. (2017) verificaram também que o treinamento resistido de baixa intensidade (30\% de 1RM) e com oclusão vascular quando comparado com o treinamento resistido tradicional $(70 \%$ de $1 \mathrm{RM})$ possui resultados similares para a redução da dor em atividades cotidianas e aumentos da foça muscular no quadríceps em indivíduos com síndrome patelar femoral. Fato importante verificado por Vasileios, Rodney e Konstantinos (2018) demonstrando que uma única sessão 
aguda de treino de baixa carga e com oclusão vascular reduziu a dor anterior no joelho e seu efeito perdurou por 45 minutos. Demonstrando que uma única sessão com restrição do fluxo sanguíneo pode ser um método seguro em induzir hipoalgesia e com isso permitir exercícios de maior intensidade realizados terapeuticamente.

O programa de reabilitação ou fisioterapia realizado com oclusão vascular para indivíduos com osteoartrite (BRYK et al., 2016; FERRAZ et al., 2017; SEGAL et al., 2015; GILES et al., 2017; SEGAL; DAVIS; MIKESKY, 2015; VASILEIOS; RODNEY; KONSTANTINOS, 2018) pode ser adaptado em situações práticas. A seguir o detalhamento de uma sugestão de um programa de reabilitação com oclusão vascular baseado em evidências:

- Frequência de 3 vezes por semana.

- Carga equivalente a 30\% de 1RM.

- Alongamento do quadríceps, 3 repetições com duração de 30 segundos.

- Ponte com contrações isométricas dos músculos do transverso do abdômen, 3 repetições de 3 segundos.

- Abdução de quadril com carga (decúbito lateral), 3 séries de 10 repetições com carga referente a $70 \%$ de $1 \mathrm{RM}$.

- Adução de quadril com carga, 3 séries de 10 repetições com carga referente a $70 \%$ de 1 RM.

- Flexão plantar, 3 séries de 10 repetições.

- Treinamento proprioceptivo, 3 séries de 10 repetições

- Cadeira extensora usando o método oclusão vascular, 3 séries de 30 repetições.

- Leg press usando o método oclusão vascular, 4 séries de 15 repetições.

Exercícios realizados dentro da água e com oclusão vascular também demonstraram efeitos importantes sobre os ganhos de força muscular e funcionalidade em mulheres com idade média de 53 anos após 8 semanas de treinamento (ARAÚJO et al., 2015).

Algo importante para se destacar durante os treinos com oclusão vascular é que a percepção subjetiva de esforço será superior quando comparado com os treinos tradicionais e sem oclusão vascular (ABE et al., 2009; OHTA et al., 2003; YASUDA et al., 2010; WEATHERHOLT et al., 2013). Por isso, deve ser abordado e explicado ao aluno antes do treinamento. 


\section{REFERÊNCIAS BIBLIOGRÁFICAS}

ABE, T. et al. Skeletal muscle size and strength are increased following walk training with restricted leg muscle blood flow: implications for training duration and frequency. International Journal of KAATSU Training Research, v. 5, n. 1, p. 9-15, 2009.

ABE, Takashi; KEARNS, Charles F.; SATO, Yoshiaki. Muscle size and strength are increased following walk training with restricted venous blood flow from the leg muscle, Kaatsu-walk training. Journal of applied physiology, v. 100, n. 5, p. 1460-1466, 2006.

ARANGIO, George A. et al. Thigh muscle size and strength after anterior cruciate ligament reconstruction and rehabilitation. Journal of Orthopaedic \& Sports Physical Therapy, v. 26, n. 5, p. 238-243, 1997.

ARAÚJO, Joamira P. et al. The effects of water-based exercise in combination with blood flow restriction on strength and functional capacity in post-menopausal women. Age, v. 37, n. 6, p. 110, 2015.

BORDE, Ron; HORTOBÁGYI, Tibor; GRANACHER, Urs. Dose-response relationships of resistance training in healthy old adults: a systematic review and metaanalysis. Sports medicine, v. 45, n. 12, p. 1693-1720, 2015.

BRYK, Flavio Fernandes et al. Exercises with partial vascular occlusion in patients with knee osteoarthritis: a randomized clinical trial. Knee Surgery, Sports Traumatology, Arthroscopy, v. 24, n. 5, p. 1580-1586, 2016.

COOK, Summer B. et al. Blood flow restricted resistance training in older adults at risk of mobility limitations. Experimental gerontology, v. 99, p. 138-145, 2017.

COOK, Summer B. et al. Skeletal muscle adaptations following blood flow-restricted training during 30 days of muscular unloading. Journal of Applied Physiology, v. 109, n. 2, p. 341-349, 2010.

DE BASTOS, Marcos et al. Tromboprofilaxia: recomendações médicas e programas hospitalares. Revista da Associação Médica Brasileira, v. 57, n. 1, p. 88-99, 2011.

ESCAMILLA, Rafael et al. Cruciate ligament force during the wall squat and the oneleg squat. Medicine \& Science in Sports \& Exercise, v. 41, n. 2, p. 408, 2009.

ESCAMILLA, Rafael F. et al. ACL Strain and Tensile Forces for Weight Bearing and Non-Weight-Bearing Exercises After ACL Reconstruction: A Guide to Exercise Selection. Journal of orthopaedic \& sports physical therapy, v. 42, n. 3, p. 208-220, 2012.

ESCAMILLA, Rafael F. et al. Effects of technique variations on knee biomechanics during the squat and leg press. Medicine \& Science in Sports \& Exercise, v. 33, n. 9, p. 1552-1566, 2001.

FERRAZ, Rodrigo Branco et al. Benefits of Resistance Training with Blood Flow Restriction in Knee Osteoarthritis. Medicine and science in sports and exercise, 2017. 
GENTIL, Paulo; BOTTARO, Martim. Influence of supervision ratio on muscle adaptations to resistance training in nontrained subjects. The Journal of Strength \& Conditioning Research, v. 24, n. 3, p. 639-643, 2010.

GILES, Lachlan et al. Quadriceps strengthening with and without blood flow restriction in the treatment of patellofemoral pain: a double-blind randomised trial. $\mathrm{Br} J$ Sports Med, v. 51, n. 23, p. 1688-1694, 2017.

IVERSEN, Erik; RØSTAD, Vibeke; LARMO, Arne. Intermittent blood flow restriction does not reduce atrophy following anterior cruciate ligament reconstruction. Journal of Sport and Health Science, v. 5, n. 1, p. 115-118, 2016.

LÓPEZ, José A.; KEARON, Clive; LEE, Agnes YY. Deep venous thrombosis. ASH Education Program Book, v. 2004, n. 1, p. 439-456, 2004.

LOPRESTI, CHARLES et al. Quadriceps insufficiency following repair of the anterior cruciate ligament. Journal of Orthopaedic \& Sports Physical Therapy, v. 9, n. 7, p. 245249, 1988.

MAZZETTI, Scott A. et al. The influence of direct supervision of resistance training on strength performance. Medicine and Science in Sports and Exercise, v. 32, n. 6, p. $1175-1184,2000$.

NAGELLI, Christopher V.; HEWETT, Timothy E. Should return to sport be delayed until 2 years after anterior cruciate ligament reconstruction? Biological and functional considerations. Sports Medicine, v. 47, n. 2, p. 221-232, 2017.

OHTA, Haruyasu et al. Low-load resistance muscular training with moderate restriction of blood flow after anterior cruciate ligament reconstruction. Acta Orthopaedica Scandinavica, v. 74, n. 1, p. 62-68, 2003.

RHEA, Matthew R. et al. A meta-analysis to determine the dose response for strength development. Medicine \& Science in Sports \& Exercise, v. 35, n. 3, p. 456-464, 2003.

SEGAL, Neil A. et al. Efficacy of blood flow-restricted, low-load resistance training in women with risk factors for symptomatic knee osteoarthritis. $P M \& R$, v. 7, n. 4, p. 376-384, 2015.

SEGAL, Neil; DAVIS, Maria D.; MIKESKY, Alan E. Efficacy of blood flow-restricted low-load resistance training for quadriceps strengthening in men at risk of symptomatic knee osteoarthritis. Geriatric orthopaedic surgery \& rehabilitation, v. 6, n. 3, p. 160-167, 2015 .

TAKARADA, Yudai; TAKAZAWA, Haruo; ISHII, Naokata. Applications of vascular occlusion diminish disuse atrophy of knee extensor muscles. Medicine \& Science in Sports \& Exercise, v. 32, n. 12, p. 2035-2039, 2000.

TENNENT, David J. et al. Blood flow restriction training after knee arthroscopy: a randomized controlled pilot study. Clinical Journal of Sport Medicine, v. 27, n. 3, p. 245-252, 2017. 
VASILEIOS, Korakakis; RODNEY, Whiteley; KONSTANTINOS, Epameinontidis. Blood Flow Restriction induces hypoalgaesia in recreationally active adult male anterior knee pain patients allowing therapeutic exercise loading. Physical Therapy in Sport, 2018. WEATHERHOLT, Alyssa et al. Modified Kaatsu training: adaptations and subject perceptions. Medicine \& Science in Sports \& Exercise, v. 45, n. 5, p. 952-961, 2013.

YASUDA, Tomohiro et al. Effect of KAATSU training on thigh muscle size and safety for a patient with knee meniscectomy over 3 years. International Journal of KAATSU Training Research, v. 13, n. 1, p. 11-14, 2017.

YASUDA, Tomohiro et al. Venous blood gas and metabolite response to low-intensity muscle contractions with external limb compression. Metabolism-Clinical and Experimental, v. 59, n. 10, p. 1510-1519, 2010.

YOW, Bobby G. et al. Blood flow restriction training after achilles tendon rupture. The Journal of Foot and Ankle Surgery, v. 57, n. 3, p. 635-638, 2018. 